\title{
Atypische Mykobakterien als Ursache von Bronchiektasen?
}

\author{
F. C. Ringshausen et al. haben in einer nationalen Studie die \\ Häufigkeit von Erkrankungen, die mit atypischen Mykobakterien \\ (PNTM: pulmonary non-tuberculous mycobacteria) assoziiert
} sind und zu einer Hospitalisation führen, systematisch analysiert. Die Studie hat gezeigt, dass PNTM gerade bei Patienten mit Zystischer Fibrose (CF) und chronisch obstruktiver Lungenerkrankung (COPD) klinisch wichtig sind und an Häufigkeit zunehmen. BMC Infect Dis 2013; 13: 231

Infektionen mit atypischen nichttuberkulösen Mykobakterien, vor allem mit M. avium intracellulare complex, M. chelonae und $M$. abscessus, nehmen sowohl bei CF- als auch bei Nicht-CF-Patienten kontinuierlich zu. Durch ihre Resistenz gegenüber zahlreichen Antibiotika sind PNTM eine klinisch-therapeutische Herausforderung. Das Forscherteam um Ringshausen von der MHH Hannover analysierte nun systematisch die Häufigkeit von PNTM in Deutschland und untersuchte bei welchen pulmonalen Krankheitsbildern diese eine Rolle spielen. Im Beobachtungszeitraum von 2005-2011 wurden hierfür 5959 Fälle von PNTM-Infektionen, die zu einer Hospitalisierung führten, herangezogen. Die Ergebnisse der Studie haben gezeigt, dass PNTM nicht nur bei CF oder Immundefekten auftreten, sondern gerade bei Patienten mit COPD an Bedeutung gewinnen. $\mathrm{Zu}$ dem nimmt die Häufigkeit durch eine Erkrankung hospitalisiert zu werden, die mit PNTM assoziiert ist, gerade bei der weiblichen Bevölkerung am deutlichsten zu. Doch während PNTM bei COPD am häufigsten mit Hospitalisationen assoziiert sind, nehmen in Deutschland Krankenhausaufenthalte aufgrund von Infektionen mit tuberkulose-typischen Mykobakterien tendenziell eher ab. Hospitalisationen mit PNTM nahmen im Beobachtungszeitraum außerdem für die Diagnosen primäre Immundefekte und AIDS ab, wohingegen auch bei CF-Patienten die Häufigkeit und Relevanz der atypischen Mykobakterien zugenommen hatte.

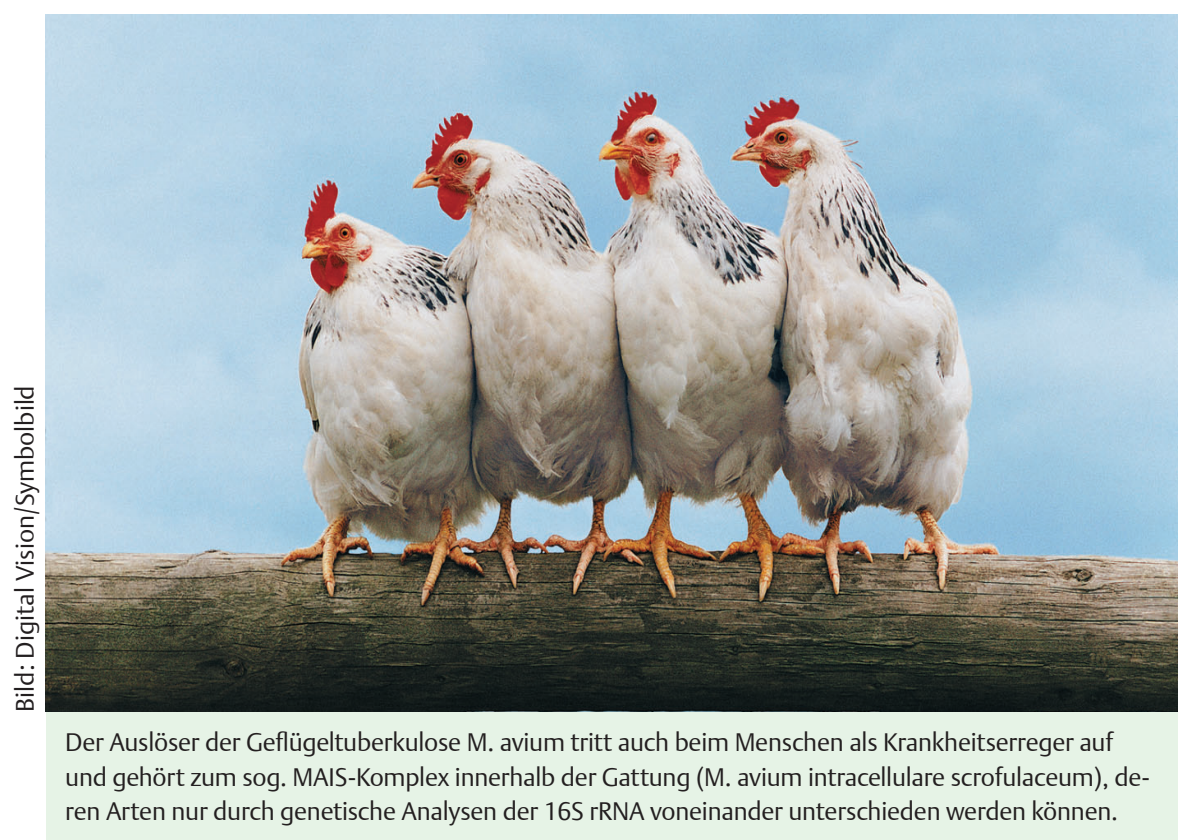

\section{Kommentar zur Studie \\ Ein Schwachpunkt dieser Studie ist die retrospektive Auswertung basierend auf ICD-Diagnosekodierungen ohne detail- liertere klinische Angaben und ohne An- gaben zu mikrobiologischen Befunden. Allerdings ist eine nationale epidemio- logisch-statistische Auswertung von dieser Größenordnung realistisch zu- nächst nur auf dieser Basis möglich. Dennoch ist diese Studie wichtig, da sie zeigt, dass atypische Mykobakterien kei- ne Rarität seltener Immundefekte sind, sondern bei der „Volkskrankheit“ COPD eine zunehmende Rolle spielen. Ange- passte Diagnose- und Therapiekonzepte sind wichtig, um diesen oft multiresis- tenten, säurefesten Bakterien erfolg- reich begegnen zu können.}

Prof. Dominik Hartl, Tübingen

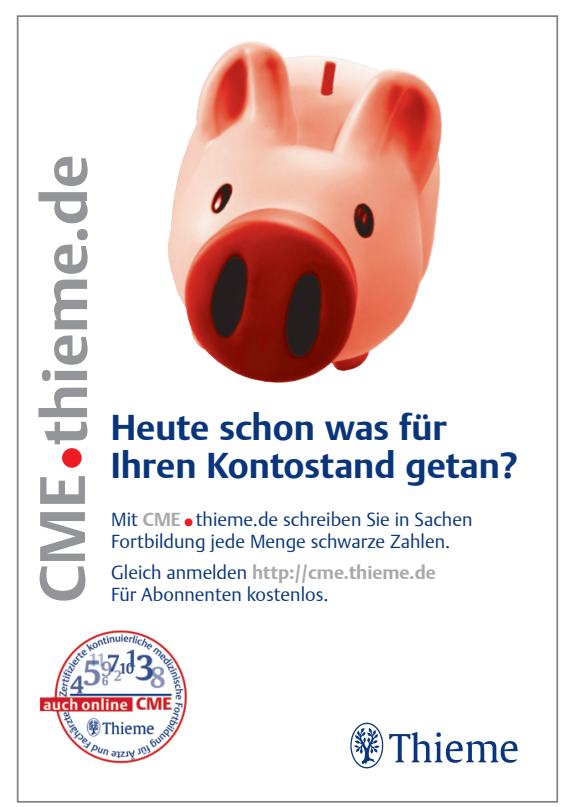

